\title{
Introduction to Special Issue on SPAA'15
}

This special issue contains revised versions of four papers that were originally presented at the 27th ACM Symposium on Parallelism in Algorithms and Architectures (SPAA'15), held on June 1315, 2015, in Portland, Oregon, as part of the Federated Computing Research Conference (FCRC'15). SPAA was sponsored by the ACM Special Interest Groups on Algorithms and Computation Theory (SIGACT) and Computer Architecture (SIGARCH) and organized in cooperation with the European Association for Theoretical Computer Science (EATCS). Financial support was provided by Akamai, Intel, Oracle Labs, SIGACT, and SIGARCH. After the conference, several papers that received excellent reviews were selected and their authors were invited to extend and resubmit their papers for consideration for this special issue of Transactions on Parallel Computing (TOPC). The following articles were selected from that group after rigorous peer-reviewing according to TOPC standards.

When processing large graphs in distributed, streaming, or MapReduce frameworks, a parallel program can often only use a sublinear amount of space. The article "Access to Data and Number of Iterations: Dual Primal Algorithms for Maximum Matching under Resource Constraints" by Kook Jin Ahn and Sudipto Guha describes a sampling-based approximation algorithm to calculate a weighted maximum matching in sublinear space and small number of rounds.

In data structures that allow unsynchronized reads-queries that make no changes to the data structures-one important problem is that a reader may be accessing a node long after it has been logically deleted from the data structure. The article "ThreadScan: Automatic and Scalable Memory Reclamation" by Dan Alistarh, William Leiserson, Alexander Matveev, and Nir Shavit describes a new approach for concurrent memory reclamation that uses operating system signaling mechanisms to allow a thread that wants to deallocate memory to detect if some other thread is accessing this memory.

Dynamic race detection algorithms can require a large amount of time and space for unstructured parallel programs. The article "Race Detection in Two Dimensions" by Dimitar Dimitrov, Martin Vechev, and Vivek Sarkar provides a space- and time-efficient race-detection algorithm for parallel programs whose control dependences can be represented as 2-D lattices-this result generalizes previous results that constrained the dependence structure to be series-parallel.

Most race-detection algorithms declare a race if a shared object has a write-write or a read-write conflict; this approach is incorrect for languages that have reducers since reduction mechanism is specifically designed to allow shared objects that can be modified concurrently in a disciplined manner. The article "Efficient Race Detection for Reducer Hyperobjects" by I-Ting Angelina Lee and Tao B. Schardl provides a race-detection algorithm especially designed for the reducers used in Cilk. This algorithm is asymptotically efficient and also efficient in practice.

We would like to thank the numerous people who made this special section possible, especially the authors for their extra work in extending their papers and carefully taking into account the reviewers' feedback, the reviewers for their thoughtful comments, Editor-in-Chief Phil Gibbons for suggesting this special section, the ACM publication staff for making the whole process run

2018 Copyright is held by the owner/author(s).

2329-4949/2018/07-ART16

https://doi.org/10.1145/3226041 
smoothly, the original reviewers from SPAA 2015, the SPAA 2015 program committee that helped select the original group of papers, and the entire SPAA organizing committee.

Kunal Agrawal Washington University in St. Louis

I-Ting Angelina Lee Washington University in St. Louis

Michael Spear Lehigh University

Guest Editors 